DOI: $10.7819 /$ rbgn.v16i51.1802

Área Temática: Finanças e Economia

\title{
Modelos Multivariados na Previsão do Valor em Risco de Carteiras de Investimento: da crise das empresas tecnológicas à crise financeira global
}

\author{
Multivariate Models to Forecast Portfolio Value at Risk: from the Dot-Com crisis to \\ the global financial crisis
}

Modelos multivariados en la previsión del valor en riesgo de carteras de inversión:
de la crisis de las empresas tecnológicas a la crisis financiera global

Vítor Manuel de Sousa Gabriel ${ }^{1}$

Recebido em 19 de novembro de 2013 / Aprovado em 22 de maio de 2014

Editor Responsável: André Taue Saito, Dr.

Processo de avaliação: Double Blind Review

\section{RESUMO}

Neste estudo é analisado o risco de mercado de uma carteira de investimento internacional por meio de uma nova proposta metodológica, baseada no Value-at-Risk, recorrendo à matriz de covariâncias de modelos multivariados do tipo GARCH e à teoria dos valores extremos, para perceber se uma estratégia de diversificação internacional minimiza o risco de mercado, assim como para verificar se a metodologia VaR capta adequadamente esse mesmo risco, aplicando testes de validação de performance. Para o efeito, foram selecionados 12 índices bolsistas internacionais, representativos de cerca de $62 \%$ da capitalização bolsista mundial, e escolhido o período compreendido entre a crise Dot-Com e a atual crise financeira global. Os resultados obtidos mostram que a proposta metodológica é uma boa alternativa na acomodação da elevada turbulência dos mercados, podendo ser considerada como uma ferramenta válida na gestão do risco de carteiras de investimento.

Palavras-chave: Mercados bolsistas. Value-at-Risk. Modelos multivariados GARCH. Teoria dos valores extremos. Backtesting.

\begin{abstract}
This study analyzed market risk of an international investment portfolio by means of a new methodological proposal based on Value-at-Risk, using the covariance matrix of multivariate GARCH-type models and the extreme value theory to realize if an international diversification strategy minimizes market risk, and to determine if the VaR methodology adequately captures market risk, by applying Backtesting tests. To
\end{abstract}

1. Mestre em Economia Financeira pela Universidade da Beira Interior, Portugal. Professor do Departamento de Gestáo e Economia do Instituto Politécnico da Guarda, Portugal. UDI -Unidade de Investigação para o Desenvolvimento do Interior, PEst-OE/EGE/UI4056/2011 - Projeto financiado pela Fundação para a Ciência e Tecnologia (FCT), Ministério da Educação e Ciência, Instituto Politécnico da Guarda (Portugal). [vigab@ipg.pt]

Endereço do autor: Instituto Politécnico da Guarda - Av. Dr. Francisco Sá Carneiro, nº50, Cep. 6300-559 - Guarda, Portugal 
this end, we considered twelve international stock indexes, accounting for about $62 \%$ of the world stock market capitalization, and chose the period from the Dot-Com crisis to the current global financial crisis. Results show that the proposed methodology is a good alternative to accommodate the high market turbulence and can be considered as an adequate portfolio risk management instrument.

Keywords: Stock markets. Value at Risk. Multivariate GARCH models. Extreme value theory. Backtesting.

\section{RESUMEN}

Este estudio analiza el riesgo de mercado de una cartera de inversiones internacionales a través de una nueva propuesta metodológica en torno al valor en riesgo, usando la matriz de covarianza de modelos multivariados GARCH y la teoría de valores extremos, para ver si una estrategia de diversificación internacional minimiza el riesgo de mercado, así como para observar si la metodología VaR capta adecuadamente el riesgo de mercado, aplicando pruebas de validación. Con este fin, se consideraron doce índices bursátiles internacionales que representan aproximadamente el $62 \%$ de la capitalización bursátil mundial, eligiéndose el período entre las crisis Dot.com y financiera mundial. Los resultados muestran que la metodología propuesta puede ser utilizada para acomodar la alta turbulencia de los mercados, y que puede ser considerada como una herramienta válida en la gestión del riesgo de las carteras de inversión.

Palabras clave: Mercados de valores. Valor en riesgo. Modelos multivariados GARCH. Teoría de los valores extremos. Backtesting.

\section{INTRODUÇÃO}

A quantificação e a diversificação do risco têm merecido uma especial atençáo por parte de investigadores e de profissionais de mercado. A publicação, em 1952, do artigo de Harry Markowitz com o título "Portfolio Selection" deu origem à teoria da carteira (MARKOWITZ, 1952). Essa teoria permite a seleção de carteiras ótimas, no pressuposto de que os investidores racionais e avessos ao risco desejam maximizar a rentabilidade da carteira, face a um determinado nível de risco. Markowitz demonstrou que um investidor pode reduzir o risco (a variância) se selecionar ativos que não descrevam movimentos idênticos, ou seja, se incluir em sua carteira ativos que não sejam altamente correlacionados. A proposta feita por Markowitz, de considerar a variância das rentabilidades dos ativos financeiros como medida de risco, manteve uma abrangência universal até finais da década de 1980. A ocorrência de diversas situaçôes de mercado, como o crash bolsista de 1987, a falência do Banco Barrings, o aumento da volatilidade dos mercados bolsistas e o investimento nos mercados emergentes, entre outras, aumentou a necessidade de desenvolver medidas de risco que expressassem as perdas potenciais.

A assinatura do Acordo de Basileia, em 1988, reforçou o propósito de padronizar normas de avaliaçấo da solidez das instituiçôes financeiras e de estabelecer níveis de capitais próprios mínimos obrigatórios, em função da exposição ao risco de crédito de cada instituição, de modo a minimizar os riscos bancários e a permitir análises comparativas internacionais entre as instituiçôes bancárias. Em 1996, viria a ser assinado o segundo acordo, conhecido por Basileia II, que teve como objetivo central a questáo do risco de mercado, e que levou à obrigatoriedade de as instituiçóes financeiras manterem determinados níveis de capital prudencial, de modo a absorverem perdas potenciais e a evitarem sua falência, garantindo também a estabilidade econômica (MANGANELLI; ENGLE, 2001). Com esse novo acordo, as instituiçôes financeiras passaram a poder dispor de autorização para adotar modelos internos de avaliação dos riscos financeiros e de cálculo do capital próprio, mediante validação e controle por parte das entidades de supervisão. Em 2010, foi assinado um novo acordo, que ficou conhecido por Basileia III, com o objetivo de reformar o sistema bancário e as maiores instituições de crédito, 
apontadas como as principais responsáveis pela crise iniciada em 2007. Esse acordo introduziu algumas mudanças ao segundo acordo, designadamente ao nível do aumento das exigências de capital das instituições bancárias e da introdução de padrôes de liquidez e de alavancagem.

A necessidade de investidores e profissionais de mercado disporem de medidas de risco, que traduzissem a probabilidade de perdas potenciais nos investimentos, deu origem a novas abordagens de gestão do risco. A gestão moderna do risco deu um significativo passo com a apresentação da metodologia Value-at-Risk (VaR), em 1993, e da metodologia Riskmetrics, em 1994. No início de 1996, o Comitê de Basileia viria a considerar a utilização da metodologia VaR como uma medida do risco de mercado, tendo autorizado as instituiçóes bancárias a utilizarem modelos internos para sua estimação. Essa metodologia revolucionou a gestáo do risco, tornando-se uma espécie de benchmark na análise e gestão do risco de mercado e fornecendo uma estimativa da perda potencial máxima em que os investidores incorrem, em função da exposição total de suas posiçôes de investimento (JORION, 2007).

Grande parte dos estudos que pressupóem a estimação do VaR recorre a modelos de heterocedasticidade condicionada, vulgarmente conhecidos como modelos da família ARCH, em resultado do trabalho de Engle (1982), os quais permitem acomodar os efeitos de clusters de volatilidade e de assimetria. So e Yu (2006), Wu e Shieh (2007) e Niguez (2008) aplicaram essa abordagem, considerando análises univariadas.

Os modelos multivariados de heterocedasticidade condicionada sáo apresentados na literatura como uma alternativa de análise do risco de mercado de carteiras de investimento (FERREIRA; LOPEZ, 2005). Sua utilização, porém, tem sido, claramente, menos frequente do que a dos modelos univariados. Dos trabalhos que recorreram a análises multivariadas destacam-se os de Morimoto e Kawasaki (2008) e de Caporin e McAleer (2012). Os primeiros compararam a performance de diversos modelos multivariados do tipo GARCH, designadamente o VECH, o BEKK, o CCC e o DCC, para as distribuições normal e t-student, na previsão do VaR de uma carteira de investimento, formada por um conjunto alargado de ativos da bolsa de Tóquio, tendo concluído que o modelo GARCH-DCC apresentou a melhor performance na previsão do VaR. Os segundos autores recorreram, igualmente, a diversos modelos multivariados do tipo GARCH, para concluírem que a performance dos modelos depende do período amostral e do tipo de carteira.

De acordo com diversos autores, entre os quais McNeil e Frey (2000), Marimoutou, Raggad, Trabelsi (2009), Assaf (2009) e Andreev et al. (2009), a Teoria dos Valores Extremos (TVE) revela boa capacidade para acomodar a ocorrência de observaçôes extremas. Não são conhecidos, contudo, estudos que considerem, no mesmo plano, os modelos multivariados do tipo GARCH e a TVE, para acomodar situaçóes de elevada turbulência.

Com a presente investigação, pretende-se expandir a literatura de finanças existente, em termos empíricos e metodológicos, recorrendo a vários modelos multivariados de heterocedasticidade condicionada e à TVE, para estimar o VaR, de modo a acomodar mais adequadamente situaçóes de elevada turbulência nos mercados, designadamente as vividas durante as crises Dot-Com e Financeira Global. Por outro lado, e de forma diferente face aos trabalhos antes referenciados, que incluíram um conjunto de ativos de um determinado mercado, no presente trabalho considera-se uma carteira de investimento diversificada internacionalmente, composta por índices representativos de diversas geografias e níveis de desenvolvimento, em particular índices representativos dos estados europeus sob assistência financeira internacional, com o objetivo de formar uma conclusão sólida acerca da capacidade desses modelos na gestão do risco de carteiras de investimento internacionais, em um contexto de elevada volatilidade e de turbulência.

Para a estimação do VaR, serão consideradas as abordagens Delta-Normal, GARCH-VECH, GARCH-CCC e GARCH-BEKK, pressupondo a distribuição normal e a distribuição t-student. Além disso, serâo considerados esses modelos, incorporando, por um lado, o 
pressuposto de efeito assimétrico, por meio da especificação Threshold GARCH (TGARCH), e por outro a TVE.

O presente estudo segue a seguinte estrutura: a parte 2 apresenta a metodologia escolhida; a parte 3 apresenta a informação acerca dos dados e os resultados empíricos, ao passo que a parte 4 expóe, de forma resumida, as principais conclusôes.

\section{PROCEDIMENTOSMETODOLÓGICOS}

\section{I Value at risk}

De acordo com Best (1998), Value at Risk é o montante máximo que se espera perder, em um ativo ou em uma carteira de ativos, durante um determinado período e para um determinado nível de confiança.

Em termos formais, o VaR pode ser definido do seguinte modo:

$\mathrm{P}\left[€ L_{t}>€ V a R_{t}\right]=\alpha$

em que $(1-\alpha)$ traduz o nível de confiança e $L$ a perda, ou seja, a variação no valor da carteira.

O VaR também pode também ser definido em termos da distribuição da taxa de rentabilidade geométrica da carteira. Considerando a probabilidade $\alpha$ e assumindo que as rentabilidades de um ativo ou de uma carteira, $\mathrm{R}_{P F}$, seguem uma distribuição normal, com média zero e desvio padrão $\sigma_{P F, t+1}$, vem $\operatorname{VaR}_{t+1}^{\alpha}=-\sigma_{P F, t+1} * \Phi_{\alpha}^{-1}$.
De acordo com Jorion (2007), Tsay (2005) e Esch, Kieffer, Lopez (2005), a distribuição t-student reúne, contudo, maior consenso para descrever o comportamento dos ativos financeiros. A superioridade dessa distribuição tem sido reportada por diversos autores, em diversos contextos e em diversos estudos empíricos (ANGELIDIS, BENOS, DEGIANNAKIS, 2004; GIOT, LAURENT, 2003). Quando as rentabilidades de uma carteira de ativos são descritas pela distribuição t-Student, o VaR é dado pela expressão (CHRISTOFFERSEN, 2003):

$\operatorname{VaR}_{t+1}^{\alpha}=-\sigma_{P F, t+1} \sqrt{\frac{d-2}{d}} t_{\alpha}^{-1}(d)$

em que $t_{\alpha}^{-1}(d)$ é o quantil à esquerda de $\alpha$, da distribuição t-student, com $d$ graus de liberdade.

A rentabilidade da carteira, $\mathrm{R}_{P F_{t+1}}$, no dia $t+1$, é determinada recorrendo à seguinte equação:

$R_{P F, t+1}=\sum_{i=1}^{n} w_{i} R_{i, t+1}$

em que $R_{i, t+1}$ é a rentabilidade associada ao índice $i$, no dia $t+1, n$ é o número de índices que compóem a carteira e $w_{i}$ é o peso atribuído ao índice $i$. Desse modo, a rentabilidade da carteira é função dos pesos relativos atribuídos aos índices e das rentabilidades de cada um dos índices.

A variância da carteira é

$\sigma_{P F, t+1}^{2}=\sum_{i=1}^{n} \sum_{j=1}^{n} w_{i} w_{j} \sigma_{i j, t+1}=\sum_{i=1}^{n} \sum_{j=1}^{n} w_{i} w_{j} \sigma_{i j, t+1} \sigma_{j, t+1} \rho_{i j, t+1}$

em que $\sigma_{i j, t+1}$ e $\rho_{i j, t+1}$ são a covariância e a correlação, respectivamente, entre o ativo $i$ e $j$, no dia $t+1$. Tendo em conta que $\sigma_{i j, t+1}=\sigma_{j i, t+1}$ e que $\rho_{i j, t+1}=$ $\rho_{j i, t+1}$, para todo o $i$ e $j$, e ainda que $\rho_{i i, t+1}=1 \mathrm{e}$ $\rho_{i j, t+1}=\rho_{j i, t+1}$, para todo o $i$ e $j, \operatorname{com} \rho_{i i, t+1}=1$ e $\sigma_{i, t+1}=\sigma_{i, t+1}^{2}$, para todo o $i$, vem:
$\sigma_{P F t+1}^{2}=w^{\prime} \sum_{t+1} w$

$\mathrm{Na}$ expressão anterior, $w$ é o vetor dos pesos da carteira e $\sum_{t+1}$ é a matriz de covariâncias das rentabilidades. No caso de $n=12$, vem: 
Modelos Multivariados na Previsão do Valor em Risco de Carteiras de Investimento: da crise das empresas tecnológicas à crise financeira global

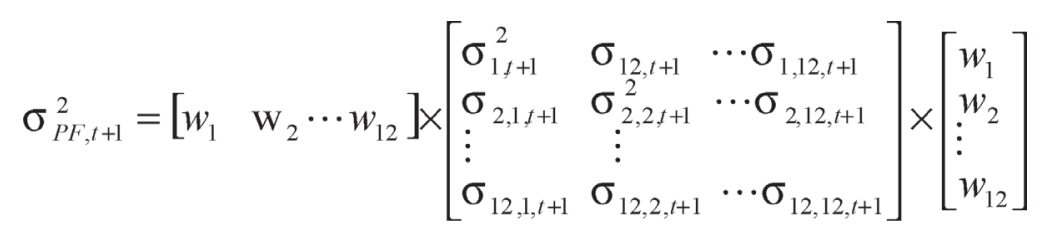

Relativamente às ponderações dos índices, neste trabalho considerou-se que elas são constantes ao longo do tempo e repartidas equitativamente, pelo que o peso relativo de cada índice corresponde a $1 / 2$ do total da carteira.

Quando se pressupóe a normalidade das rentabilidades, a metodologia atrás apresentada é designada por Delta Normal, e o VaR da carteira é dado pela expressão:

$$
\operatorname{VaR}_{t+1}^{\alpha}=-\sigma_{P F, t+1} * \Phi_{\alpha}^{-1}
$$

Com o objetivo de incorporar a heterocedasticidade condicionada, habitualmente presente nos índices de mercados bolsistas, serão estimadas matrizes de variância-covariância, recorrendo a diversos modelos GARCH multivariados, designadamente os modelos VECH, BEKK e CCC, cujos pressupostos teóricos apresentamos no ponto seguinte.

\subsection{Modelos multivariados de heterocedas- ticidade condicionada}

\section{Modelo VECH}

O modelo GARCH-VECH foi proposto por Bollerslev, Engle, Wooldrigde (1988), podendo ser escrito da seguinte forma:

$$
\operatorname{vech}\left(H_{t}\right)=C+\sum_{j=1}^{q} A_{j} \operatorname{vech}\left(\varepsilon_{t-1} \varepsilon_{t-1}^{\prime}\right)+\sum_{j=1}^{p} B_{j} \operatorname{vech}\left(H_{t-j}\right)
$$

em que $H_{t}$ diz respeito à matriz de variâncias e covariâncias condicionais. De acordo com Scherrer e Ribarits (2007), a compreensão da matriz torna-se mais fácil quando ela inclui mais do que duas variáveis, como é o caso estudado. A representação do modelo VECH é baseada na assunção de que a variância condicional depende do quadrado dos resíduos desfasados e a covariância condicional depende dos resíduos cruzados e desfasados e das covariâncias desfasadas de outras séries (HARRIS; SOLLIS, 2003). Na equação do modelo, $A$ e $B$ são matrizes de coeficientes, de tamanho $1 / 2 N(N+1) \times 1 / 2 N(N+1), C$ é um vetor de termos constantes, de tamanho $1 / 2 N(N+1) \times 1$, e $p$ e $q$ indicam o número de ordem dos modelos GARCH e ARCH, respectivamente.

\section{Modelo de Correlação Condicional Constante (CCC)}

O modelo CCC foi proposto por Bollerslev (1990), implicando que o pressuposto de correlação condicional seja constante no período $t$. A matriz de covariância no modelo é dada por:

$H_{t}=D_{t} \mathrm{RD}_{t}$

em que $H_{t}$ é a matriz de covariância, $D_{t}$ é a matriz diagonal, cujos elementos são compostos pela variância condicional, $h_{i j, t}$ de cada série, no momento $t$, e $R$ é a matriz dos coeficientes de correlação linear constante, $p_{i j, t}$.

Supondo a distribuição normal condicional, o coeficiente de correlação linear constante, entre as séries $i$ e $j$, é dado por:

$\hat{p}_{\ddot{j}}=\sum_{t=0}^{T} \hat{e}_{i, t} \hat{e}_{j, t}\left(\sum_{t=0}^{T} \hat{e}_{i, t}^{2}\right)^{-1 / 2}\left(\sum_{t=0}^{T} \hat{e}_{j, t}^{2}\right)^{-1 / 2}$

em que $\hat{e}_{i, t}$ e $\hat{e}_{j, t}$ são resíduos padronizados das séries $i$ e $j$, obtidos por meio de estimação univariada, recorrendo a modelos da família GARCH. Para se obter a matriz de covariâncias, estimam-se modelos univariados e calculam-se os resíduos 
padronizados, que por sua vez servem de base ao cálculo dos coeficientes de correlação linear.

Uma forma de medir a correlação passa por tratar os resíduos como os dos modelos de variância-covariância. Por definição,

$\sigma_{i j, t+1}=\sigma_{i, t+1} \sigma_{j \neq t+1} \rho_{i j, t+1}$

Deste modo,

$\rho_{i j, t+1}=\frac{\sigma_{i j, t+1}}{\sigma_{i, t+1} \sigma_{j, t+1}}$

Portanto, se, por exemplo, $\rho_{i j, t+1}=(1-\lambda) R_{i, t} R_{j, t}+\lambda \sigma_{i j, t}$, para qualquer $\mathrm{i}, \mathrm{j}$, então:

$\left.\rho_{i j, t+1}=\frac{(1-\lambda) R_{i, t} R_{j, t}+\lambda \sigma_{i j, t}}{\sqrt{\left((1-\lambda) R_{i, t}^{2}+\lambda \sigma_{i, t}^{2}\right)\left((1-\lambda) R_{j, t}^{2}+\lambda \sigma_{j, t}^{2}\right.}}\right)$

\section{Modelo BEKK}

Uma alternativa para estimar a variância condicionada é a do modelo BEKK (BABA-ENGLE-KRAFT-KRONER), proposto por Engle e Kroner (1995), que pode expresso da seguinte forma:

$$
H_{t}=C^{\prime} C+\sum_{j=1}^{q} A^{\prime}{ }_{j} \varepsilon_{t-1} \varepsilon_{t-1}^{\prime} A_{j}+\sum_{j=1}^{p} B_{j}^{\prime} H_{t-j} B_{j}
$$

O modelo BEKK garante que a matriz $H_{t}$ é semipositiva, ao contrário do que acontece no modelo $\mathrm{VECH}$, em que não há tal garantia. No modelo BEKK, $A$ e $B$ são matrizes de tamanho $N \times N, C$ é uma matriz triangular superior de coeficientes, $N$ é o número de séries consideradas no modelo, e $p$ e $q$ indicam o número de ordem dos modelos GARCH e ARCH, respectivamente.

\subsection{Teoria do valor extremo}

Um dos mais recentes métodos de cálculo do VaR baseia-se na Teoria dos Valores Extremos (TVE). Segundo diversos autores, a grande vantagem da TVE, em comparação com outras abordagens, é a de permitir um bom ajustamento às caudas da distribuição das rentabilidades (DANIELSSON, DE VRIES, 1997; EMBRECHTS, KLUPPERLBERG, MIKOSCH, 1997; MCNEIL, 1998; REISS, THOMAS, 1997).

Considere-se a probabilidade de a rentabilidade estandardizada, $z$, deduzida do limiar (threshold), $u$, ser inferior a um determinado valor $x$, tendo em conta que a rentabilidade estandardizada está acima do limiar, $u$.

$F_{u}(x) \equiv \operatorname{Pr}\{z-u \leq x \mid z>u\}$, em que $x>u$

Considerem-se, ainda, as rentabilidades estandardizadas acima de um limiar, tendo em conta que a distribuição, $F_{u}(x)$, depende da escolha do limiar. Usando a definição geral de probabilidade condicional, vem

$$
F_{u}(x)=\frac{P_{r}\{u<z \leq x+u\}}{P_{r}\{z>u\}}=\frac{F(x+u)-F(u)}{1-F(u)}
$$

Assim, a distribuição das rentabilidades estandardizadas, acima do limiar, pode ser escrita em função da distribuição das rentabilidades estandardizadas, $F(x)$.

No âmbito da TVE, à medida que os valores extremos se afastam do limiar, $u$, convergem para a distribuição generalizada de Pareto (GPD), $G(x, \xi, \beta)$. Essa distribuição é definida genericamente do seguinte modo:

$G(x, \xi, \beta)=\left\{\begin{array}{l}1-(1+\xi x / \beta)^{-1 / \xi}, \text { se } \xi \neq 0 \\ 1-\exp (-x / \beta), \text { se } \xi=0\end{array}\right.$

$\operatorname{Com} \beta>0$, e

$\left\{\begin{array}{l}x \geq u, \operatorname{se} \xi \geq 0 \\ u \leq x \leq u-\beta / \xi, \text { se } \xi<0\end{array}\right.$

em que o coeficiente de assimetria, $\xi$, é positivo e representa a velocidade de decaimento da cauda, $\beta$ é o parâmetro scale e $\mu$ o limiar. 
Modelos Multivariados na Previsão do Valor em Risco de Carteiras de Investimento: da crise das empresas tecnológicas à crise financeira global

Apoiando-nos na metodologia de McNeil (1999), considerem-se os pontos $x, \operatorname{com} x>u$, na cauda da distribuição. Fazendo $y=x+u$, vem:

$$
F_{u}(x)=\frac{F(x+u)-F(u)}{1-F(u)}
$$

Para obter

$$
F(y)=1-[1-F(u)]\left[1-F_{u}(y-u)\right]
$$

seja $T$ o tamanho da amostra total e $T_{u}$ o número de observaçóes acima do limiar, $u$. O termo $1-F(u)$ pode, então, ser estimado como a proporção de observações $\left(T_{u} / T\right)$ acima do limiar. Por sua vez, $F_{u}(*)$ pode ser obtido através do estimador de máxima verossimilhança, das observaçôes estandardizadas, acima de um limiar escolhido. Supondo que $\hat{i} \neq 0$, a distribuição vem

$F(y)=1-T_{u} / T(1+\xi(y-u) / \beta)^{-1 / \xi}$

A função de probabilidade acumulada, e igual a $1-\alpha$, em que só há uma probabilidade, $\alpha$, de obter perdas estandardizadas superiores ao valor do quantil, é implicitamente definida por:

$$
F\left(F_{1-\alpha}^{-1}\right)=1-\alpha
$$

A partir da definição de $F(*)$, pode ser resolvido para o quantil de modo a obter:

$$
F_{1-\alpha}^{-1}=\mu\left[\alpha /\left(T_{u} / T\right)\right]^{-\xi}
$$

O VaR é obtido a partir da TVE e do modelo de variância escolhido, com base na seguinte expressão:

$$
\operatorname{VaR}_{t+1}^{p}=\sigma_{P F, t+1} F_{1-p}^{-1}=\sigma_{P F, t+1} u\left[\alpha /\left(T_{u} / T\right)\right]^{\xi}
$$

em que $\alpha$ é o nível de confiança do VaR. Para estimar o VaR, com um nível de confiança $\alpha$, utiliza-se o estimador TVE e um ponto de corte $u$, de modo a considerar uma percentagem de dados, da cauda esquerda, superior a $1-\alpha$.

\subsection{Avaliação da performance dos modelos VaR}

A metodologia considerada no presente estudo, para avaliação da performance dos modelos VaR, pressupóe a contagem do número de vezes que as perdas efetivas superam as estimativas resultantes da metodologia VaR. O resultado dessa contagem, em um determinado período, pressupóe a consideração de um certo nível de confiança para estimação do modelo.

Considerando a série das rentabilidades logarítmicas diárias, $\mathrm{R}_{P F}$, e a série das previsões calculadas pelo VaR, para um determinado nível de confiança $\left(\operatorname{VaR} R^{\alpha}\right)$ e para $P\left(R_{P F, t+1}<-V a R_{t+1}^{\alpha}\right)=\alpha$, obtém-se uma sucessão binária, também designada por "sequência de Hit", em função do número de ultrapassagens do $\operatorname{VaR}_{t+1}^{\alpha}$, do seguinte modo:

$$
I_{t}=\left\{\begin{array}{l}
1, \operatorname{se} \mathrm{R}_{P F, t+1}<-\operatorname{VaR}_{t+1}^{\alpha} \\
0, \text { se } \mathrm{R}_{P F, t+1} \geq-\operatorname{VaR}_{t+1}^{\alpha}
\end{array}\right.
$$

A “sequência de Hit" apresenta o valor 1 , no dia $t+1$, se a perda naquele dia for superior ao valor do VaR previsto, antecipadamente, para esse mesmo dia. Se o VaR não for ultrapassado, então a sequência assume o valor 0 .

A partir da "sequência de Hit", são aplicados os testes de Backtesting do VaR. Entre os principais testes disponíveis, destacam-se o teste de cobertura incondicional, o teste de independência e o teste de cobertura condicional, que serão abordados nos próximos pontos.

\subsection{Teste de cobertura incondicional ou Teste de Kupiec}

O teste de cobertura incondicional (unconditional coverage test) ou teste de Kupiec (1995) envolve a contagem do número de vezes que as estimativas produzidas pelo VaR são superadas por um ativo ou por uma carteira de ativos, a partir de uma determinada amostra. A hipótese nula desse teste estabelece que a verdadeira proporção de exceções, $\pi$, é consistente com o quantil de falhas, $\alpha$, previsto pelo modelo $V a R$ : 


$$
H_{0}: E\left[I_{t}\right] \equiv \pi=\alpha
$$

A estatística teste de máxima verossimilhança para essa hipótese é:

$$
L R_{u c}=-2 \ln \left(\frac{\alpha^{T_{1}}(1-\alpha)^{T_{0}}}{\hat{\pi}^{T_{1}}(1-\hat{\pi})^{T_{0}}}\right) \sim \chi^{2}(1)
$$

em que $T_{1}$ é o número de falhas $\left(I_{t}=1\right)$, para um dado número total de dias $T\left(I_{t}\right), T_{0}$ é o número de acertos $\left(I_{t}=0\right)$, e $\hat{\pi}\left(T_{1} / T\right)$ corresponde à proporção de falhas (exceçôes).

\subsubsection{Teste de Independência}

Christoffersen (2003) desenvolveu o teste de independência para analisar a ocorrência de exceçôes em cluster, testando a hipótese nula de as exceçôes no modelo serem independentes e identicamente distribuídas (IID), ou seja, de que a probabilidade de ocorrerem exceções é igual, independentemente de ter ocorrido uma exceção no dia anterior. O teste tem como objetivo analisar o modo como ocorrem as exceçóes, no pressuposto de que quaisquer dois elementos da sequência têm de ser independentes uns dos outros. Quando essa condição não é verificada, é um indicador de que o modelo de VaR não é suficientemente sensível para acolher alteraçóes no risco de mercado (CAMPBELL, 2005).

$\mathrm{O}$ teste de independência proporciona, pois, as condiçôes estatísticas que permitem rejeitar um modelo VaR, com exceçóes em cluster. Para tal, assume-se que a "sequência de hit" é dependente ao longo do tempo e que pode ser descrita por uma sequência de Markov de primeira ordem.

A hipótese de independência $\left(\pi_{0}=\pi_{1}\right)$ pode ser testada recorrendo ao teste de verossimilhança

$$
L R_{\text {ind }}=-2 \ln \left[L(\hat{\pi}) / L\left(\hat{\Pi}_{1}\right)\right] \sim \chi_{1}^{2}
$$

em que $L(\hat{\pi})$ é a verossimilhança, sob a hipótese alternativa, a partir do teste $\mathrm{LR}_{u c}$.
A estatística teste $\mathrm{LR}_{\text {ind }}$, à independência do número de exceções consecutivas, em $t$ e $t-1$, é

$L R_{\text {ind }}=-2 \ln \left(\frac{(1-\hat{\pi})^{\left(T_{00}+T_{10}\right)} \hat{\pi}^{\left(T_{10}+T_{11}\right)}}{\left(1-\hat{\pi}_{01}\right)^{T_{00}} \hat{\pi}_{0}^{T_{01}}\left(1-\hat{\pi}_{11}\right)^{T_{10}} \hat{\pi}_{11}^{T_{11}}}\right) \sim \chi^{2}(1)$

Em amostras de pequena dimensão, é frequente a nulidade de $T_{\mathbb{1}}$. Nesses casos, a estatística $\mathrm{LR}_{\text {ind }}$ é calculada por meio de

$L R_{\text {ind }}=-2 \ln \left(\frac{(1-\hat{\pi})^{\left(T_{T_{00}}+T_{10}\right)} \hat{\pi}^{\left(T_{01}+T_{11}\right)}}{\left(1-\hat{\pi}_{01}\right)^{T_{00}} \hat{\pi}_{01}{ }^{T_{01}}}\right) \sim \chi^{2}(1)$

em que $T_{j}, i, j=0,1$ é o número de observações, com $j$ a suceder a $i$. A probabilidade de amanhã acontecer uma exceção, tendo em conta que hoje não ocorreu, é dada por $\hat{\pi}_{01}=\frac{T_{01}}{T_{00}+T_{01}}$, ao passo que a probabilidade de amanhã acontecer uma exceção condicional, dado que hoje ocorreu uma exceçáo, é dada por $\hat{\pi}_{11}=\frac{T_{11}}{T_{10}+T_{11}}$. Por sua vez, $\pi$ diz respeito à taxa de falhas ou de exceçóes registradas.

\subsubsection{Teste de cobertura condicional ou Teste de Christoffersen}

Para testar a cobertura não condicional e a independência, Christoffersen (2003) desenvolveu as já apresentadas estatísticas $\mathrm{LR}_{u c}$ e $\mathrm{LR}_{\text {ind }}$. Para testar em simultâneo as duas propriedades, o mesmo autor desenvolveu o teste de cobertura condicional (Conditional Coverage Test), $\mathrm{LR}_{c c}$, que é dado por

$$
L R_{c c}=L R_{i n d}+L R_{u c} \sim \chi^{2}(2)
$$

\section{DADOS E RESULTADOS EMPÍRICOS}

\section{I Dados e estatísticas}

Com o objetivo de analisar a performance dos modelos de gestão do risco de mercado, foram selecionados índices representativos dos mercados internacionais, concretamente índices europeus, não europeus, desenvolvidos e emergentes, de acordo com a classificação atribuída pela Morgan 
Stanley Capital International, e que representavam, em 2010, cerca de 62\% da capitalização bolsista mundial, como pode ser visto na Tabela 1. O conjunto de mercados desenvolvidos incluiu mercados europeus e não europeus. Do continente europeu, foram escolhidos os mercados da Alemanha (DAX 30), da França (CAC 40), do Reino Unido (FTSE 100), de Espanha (IBEX
35), da Irlanda (ISEQ Overall), da Grécia (ATG) e de Portugal (PSI 20). Do conjunto de mercados desenvolvidos, não europeus, foram escolhidos os EUA (Dow Jones), o Japão (Nikkei 225) e Hong Kong (Hang-Seng). Relativamente aos mercados emergentes, foram selecionados o Brasil (Bovespa) e a Índia (Sensex).

TABELA 1 - Capitalização bolsista dos mercados, em percentagem da capitalização mundial

\begin{tabular}{cccccccccccc}
\hline EUA & Reino Unido & França & Japão & Espanha & Brasil & Alemanha & Portugal & Grécia & Hong Kong & Índia & Irlanda \\
\hline 30,5 & 5,5 & 3,4 & 7,3 & 2,1 & 2,8 & 2,5 & 0,1 & 0,1 & 4,8 & 2,9 & 0,06 \\
\hline
\end{tabular}

Fonte: World Bank (c2014)

Os dados utilizados neste estudo foram obtidos junto da Econostats e cobrem o período compreendido entre 4 de outubro de 1999 e 30 de junho de 2011, no qual ocorreram duas importantes crises nos mercados bolsistas, a crise das empresas tecnológicas e a crise financeira global. O lapso temporal entre essas duas crises (2003 a 2007) correspondeu ao período de uma alta generalizada dos valores dos índices bolsistas mundiais.

As séries dos valores de fechamento dos índices foram transformadas em séries de rentabilidade, $r_{t}$, por meio da aplicação da expressão $\ln \left(P_{t} / P_{t-1}\right)$, em que $P_{t}$ e $P_{t-1}$ representam os valores de fechamento de um determinado índice nos dias $t$ e $t-1$, respectivamente.

Na Tabela 2 são apresentadas as principais estatísticas descritivas de cada uma das séries de rentabilidades dos índices, os testes de Jarque-Bera à normalidade das séries, os testes à estacionaridade e os testes LM de Engle (1982) à presença de heterocedasticidade.

A análise das estatísticas descritivas permite extrair a conclusão de que apenas seis dos 12 índices tiveram rentabilidade média diária positiva.

TABELA 2 - Estatísticas descritivas das séries

\begin{tabular}{lccccccccc}
\hline & Média & Máximo & Mínimo & Desvio-padráo & Assimetria & Curtose & $\begin{array}{c}\text { JB } \\
\text { (Prob.) }\end{array}$ & $\begin{array}{c}\text { ADF } \\
(\text { Prob. })\end{array}$ & $\begin{array}{c}\text { LM } \\
(\text { Prob. })\end{array}$ \\
\hline ATG & $-0,000513$ & 0,088129 & $-0,10214$ & 0,016668 & $-0,154716$ & 6,9734 & $(0,0000)$ & $(0,0000)$ & $(0,0000)$ \\
BOV & 0,000597 & 0,136766 & $-0,145659$ & 0,019319 & $-0,223056$ & 7,6515 & $(0,0000)$ & $(0,0000)$ & $(0,0000)$ \\
CAC & $-0,00005$ & 0,105946 & $-0,094715$ & 0,015649 & 0,042147 & 7,9632 & $(0,0000)$ & $(0,0000)$ & $(0,0000)$ \\
DAX & 0,000119 & 0,107975 & $-0,074335$ & 0,016158 & 0,061832 & 7,2076 & $(0,0000)$ & $(0,0000)$ & $(0,0000)$ \\
DJ & 0,000061 & 0,105083 & $-0,082005$ & 0,012625 & 0,031963 & 10,6597 & $(0,0000)$ & $(0,0000)$ & $(0,0000)$ \\
FTSE & $-0,000006$ & 0,093842 & $-0,092646$ & 0,013034 & $-0,105256$ & 9,1214 & $(0,0000)$ & $(0,0000)$ & $(0,0000)$ \\
HANG & 0,000191 & 0,134073 & $-0,135825$ & 0,016327 & $-0,007715$ & 10,848 & $(0,0000)$ & $(0,0000)$ & $(0,0000)$ \\
IBEX & 0,000026 & 0,134836 & $-0,108341$ & 0,015264 & 0,047132 & 9,5441 & $(0,0000)$ & $(0,0000)$ & $(0,0000)$ \\
ISEQ & $-0,000166$ & 0,097331 & $-0,139636$ & 0,01498 & $-0,652159$ & 11,0664 & $(0,0000)$ & $(0,0000)$ & $(0,0000)$ \\
NIKKEI & $-0,000205$ & 0,094941 & $-0,12111$ & 0,015462 & $-0,644815$ & 9,0135 & $(0,0000)$ & $(0,0000)$ & $(0,0000)$ \\
PSI & $-0,000113$ & 0,101959 & $-0,103792$ & 0,011677 & $-0,223954$ & 12,9958 & $(0,0000)$ & $(0,0000)$ & $(0,0000)$ \\
SENSEX & 0,000483 & 0,159901 & $-0,118092$ & 0,017047 & $-0,207255$ & 9,6325 & $(0,0000)$ & $(0,0000)$ & $(0,0000)$ \\
\hline
\end{tabular}

Fonte: Do autor. 
Com o objetivo de averiguar a não estacionaridade ou integração das séries, aplicamos o tradicional teste ADF. A hipótese nula dos testes estipula que as séries têm raiz unitária, ou seja, que as séries são integradas de ordem 1 , face à hipótese alternativa de as séries não possuírem raiz unitária. Os resultados confirmam que os valores das probabilidades dos testes das 12 séries são inferiores a $1 \%$, rejeitando-se claramente a hipótese nula de integração das séries, e concluindo-se que elas evidenciam estacionaridade ou são I (0).

Para testar a existência de heterocedasticidade condicionada (efeitos ARCH), nas taxas de rentabilidade dos índices, foram estimados processos autorregressivos de primeira ordem e aplicados testes LM de Engle (1982), para o desfasamento 20, aos resíduos dos processos autorregressivos. Em todos os casos, as probabilidades dos testes LM permitem concluir que, para o nível de significância de $1 \%$, as séries das taxas de rentabilidade dos índices exibem heterocedasticidade condicionada, pelo que a utilização de modelos do tipo GARCH se revela adequada.

\subsection{Resultados empíricos}

Todas as séries de rentabilidades, sem exceção, evidenciam sinais de desvio face à hipótese de normalidade, já que os coeficientes de assimetria e de curtose são estatisticamente diferentes dos de uma distribuição normal (0 e 3, respectivamente). Para se confirmar a adequação do ajustamento da distribuição normal às distribuiçôes empíricas das 12 séries, em todos os períodos analisados, foi também aplicado o teste de aderência de Jarque-Bera. Tendo em conta as respetivas probabilidades associadas (iguais a 0 ), concluímos que todas as séries são estatisticamente significativas a $1 \%$, rejeitando-se claramente a hipótese de normalidade das mesmas.
Para averiguar a performance dos modelos VaR, na captação do risco dos 12 mercados estudados, consideramos uma carteira teórica de investimento, ponderada equitativamente, a partir da qual foram estimados diversos modelos multivariados de heterocedasticidade condicionada, designadamente as especificaçôes VECH, BEKK e CCC, para as distribuiçôes normal e t-student, e para o efeito assimétrico, e que serviram de base às estimaçôes VaR. Por último, foi aplicada a teoria dos valores extremos, nas três especificaçôes, para os modelos GARCH e Threshold GARCH (TGARCH), de modo a incorporar o efeito assimétrico, e para as distribuiçôes normal e t-student, com o objetivo de perceber se esta responde adequadamente às variaçóes extremas que caracterizaram o período estudado. Em todos os casos referidos, as estimaçóes dos modelos $\mathrm{VaR}$ consideraram os níveis de confiança de 5\%, 1\% e $0,5 \%$. O primeiro nível de confiança segue a metodologia Riskmetrics, o segundo tem em consideração a exigência do Comitê de Basileia II, ao passo que o último foi escolhido com o objetivo de perceber a consequência de uma condição de estimação mais exigente.

Na Tabela 3 são comparados os somatórios dos $\mathrm{VaR}$ dos diversos índices com os $\mathrm{VaR}$ da carteira, para os diversos modelos de estimação e para os três níveis de confiança.

Em todos os casos comparados, o VaR da carteira é claramente inferior ao do somatório dos VaR individuais dos índices, havendo, por essa razão, motivo para acreditar que o risco de mercado possa ser reduzido pela via da diversificação. Embora as ligaçóes entre os mercados tendam a ser cada vez mais próximas, a opção por uma estratégia de investimento, assente no pressuposto de diversificação internacional, que considere um conjunto alargado de mercados, pode constituir uma forma de proteção face ao risco de mercado. 
Modelos Multivariados na Previsão do Valor em Risco de Carteiras de Investimento: da crise das empresas tecnológicas à crise financeira global

TABELA 3 - Comparação entre o var da carteira e o somatório das estimativas var dos índices

\begin{tabular}{|c|c|c|c|c|c|c|c|c|}
\hline \multirow{2}{*}{\multicolumn{3}{|c|}{$\frac{\text { Distribuiçãoo }}{\text { Normal }}$}} & \multicolumn{6}{|c|}{ Modelo } \\
\hline & & & Vech & Tvech & BEKK & TBEKK & CCC & TCCC \\
\hline \multirow{6}{*}{ 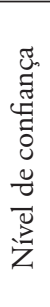 } & \multirow{2}{*}{0,005} & Somatório VaR & 0,0396 & 0,0396 & 0,0396 & 0,0395 & 0,0397 & 0,0395 \\
\hline & & VaR da carteira & 0,0279 & 0,0279 & 0,0279 & 0,0278 & 0,0280 & 0,0278 \\
\hline & \multirow{2}{*}{0,01} & Somatório VaR & 0,0358 & 0,0358 & 0,0357 & 0,0357 & 0,0358 & 0,0357 \\
\hline & & VaR da carteira & 0,0252 & 0,0252 & 0,0252 & 0,0251 & 0,0253 & 0,0251 \\
\hline & \multirow{2}{*}{0,05} & Somatório VaR & 0,0263 & 0,0263 & 0,0263 & 0,0263 & 0,0263 & 0,0263 \\
\hline & & VaR da carteira & 0,0178 & 0,0178 & 0,0178 & 0,0178 & 0,0179 & 0,0178 \\
\hline \multicolumn{3}{|c|}{ t-student } & Vech & Tvech & BEKK & TBEKK & CCC & TCCC \\
\hline \multirow{6}{*}{ 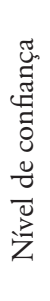 } & \multirow{2}{*}{0,005} & Somatório VaR & 0,0437 & 0,0437 & 0,0437 & 0,0442 & 0,0437 & 0,0440 \\
\hline & & VaR da carteira & 0,0308 & 0,0308 & 0,0308 & 0,0311 & 0,0308 & 0,0310 \\
\hline & \multirow{2}{*}{0,01} & Somatório VaR & 0,0381 & 0,0381 & 0,0381 & 0,0384 & 0,0381 & 0,0384 \\
\hline & & VaR da carteira & 0,0269 & 0,0269 & 0,0268 & 0,0270 & 0,0269 & 0,0270 \\
\hline & \multirow{2}{*}{0,05} & Somatório VaR & 0,0255 & 0,0254 & 0,0254 & 0,0254 & 0,0255 & 0,0256 \\
\hline & & VaR da carteira & 0,0176 & 0,0176 & 0,0176 & 0,0176 & 0,0176 & 0,0177 \\
\hline
\end{tabular}

Notas: as estimativas $\mathrm{VaR}$, apresentadas na tabela, foram obtidas a partir da matriz de variâncias-covariâncias de modelos GARCH, sem efeito assimétrico (VECH, BEKK e CCC) e com efeito assimétrico (TVECH, TBEKK e TCC), considerando os intervalos de confiança de 99,5\% (0,5), 99\% (1) e 95\% (5).

Fonte: Do autor.

A Tabela 4 apresenta as médias das estimativas VaR dos diversos modelos. A característica mais evidente é a de que os valores médios dependem mais da distribuição considerada do que da previsão da matriz de covariância. Na grande maioria dos casos, as médias das estimativas VaR, produzidas com base na distribuição t-student, são superiores às resultantes da distribuição normal, em especial nos níveis de confiança de 0,5\% e de 1\%. Apenas os modelos VECH e TVECH-TVE, nesses dois níveis de confiança, e o modelo TCCC-TVE, no nível de confiança de 0,5\%, não corroboram essa superioridade. Para o quantil mais elevado, a conclusão é contrária, revelando superioridade das estimativas baseadas na distribuição normal, em 67\% dos casos.

TABELA 4 - Estimativas médias dos modelos VaR

\begin{tabular}{|c|c|c|c|c|c|c|}
\hline & \multicolumn{3}{|c|}{ Normal } & \multicolumn{3}{|c|}{ t-student } \\
\hline & 0,005 & 0,01 & 0,05 & 0,005 & 0,01 & 0,05 \\
\hline VECH & 0,02522 & 0,02277 & 0,01610 & 0,02439 & 0,02127 & 0,01395 \\
\hline BEKK & 0,02534 & 0,02289 & 0,01618 & 0,02745 & 0,02370 & 0,01521 \\
\hline CCC & 0,02458 & 0,02220 & 0,01570 & 0,02675 & 0,02333 & 0,01530 \\
\hline TVECH & 0,02482 & 0,02241 & 0,01585 & 0,03122 & 0,02647 & 0,01636 \\
\hline TBEKK & 0,02499 & 0,02257 & 0,01596 & 0,02656 & 0,02306 & 0,01498 \\
\hline TCCC & 0,02411 & 0,02178 & 0,01540 & 0,02676 & 0,02333 & 0,01530 \\
\hline VECH-TVE & 0,03092 & 0,02522 & 0,01572 & 0,03289 & 0,02635 & 0,01575 \\
\hline BECK-TVE & 0,03409 & 0,02705 & 0,01580 & 0,03546 & 0,02766 & 0,01555 \\
\hline CCC-TVE & 0,03139 & 0,02542 & 0,01557 & 0,03179 & 0,02574 & 0,01577 \\
\hline TVECH-TVE & 0,03200 & 0,02590 & 0,01585 & 0,03189 & 0,02580 & 0,01576 \\
\hline TBEKK-TVE & 0,03068 & 0,02520 & 0,01597 & 0,03381 & 0,02677 & 0,01556 \\
\hline TCCC-TVE & 0,03048 & 0,02482 & 0,01539 & 0,03047 & 0,02494 & 0,01566 \\
\hline
\end{tabular}

Notas: as estimativas VaR, apresentadas na tabela, foram obtidas a partir da matriz de variâncias-covariâncias de modelos GARCH, sem efeito assimétrico (VECH, BEKK, CCC), com efeito assimétrico (TVECH, TBEKK e TCC) e com a TVE, considerando os intervalos de confiança de $99,5 \%(0,5), 99 \%$ (1) e $95 \%$ (5).

Fonte: Do autor. 
A Tabela 5 resume a proporção de falhas dos vários modelos de estimação, ou seja, a percentagem de vezes que as estimativas VaR foram superadas por rentabilidades diárias, em resultado da ocorrência de eventos de mercado inesperados. A análise dos resultados apresentados nesta tabela revela que os modelos que não recorrem à teoria do valor extremo, quer no pressuposto de distribuição normal quer no de distribuição t-student, de um modo geral evidenciam dificuldades de adaptação às variaçóes extremas de mercado.

No caso das estimativas assentes em modelos do tipo GARCH, só o nível de confiança mais elevado, na distribuição normal, produz uma proporção de falhas abaixo do respetivo nível de confiança. Por sua vez, na distribuição t-student, em nenhum caso tal situação se verifica, razão para se concluir que as respectivas estimativas são demasiado conservadoras para os vários níveis de confiança. Em geral, a simples consideração do efeito assimétrico não melhora significativamente a situação anterior. A incorporação da especificação de efeito assimétrico, contudo, faz que o modelo $\mathrm{VECH}$ registre uma mudança positiva, nos três quantis, com a percentagem de exceçôes a ser, em todos os casos, inferior ao respectivo nível de confiança.

O recurso à teoria dos valores extremos conduz a conclusões diversas das enunciadas anteriormente, acerca dos modelos GARCH multivariados. As estimativas dos modelos GARCH e dos modelos TGARCH, assentes nas duas distribuições, proporcionam conclusôes idênticas, com percentagens de exceçôes inferiores aos níveis de confiança de $0,5 \%$ e de $1 \%$, ao contrário do que acontece para o nível de confiança de $5 \%$. Esse fato dá uma primeira indicação da superioridade da teoria dos valores extremos face às restantes metodologias, como teremos oportunidade de confirmar com a aplicação dos testes de performance.

TABELA 5 - Proporção de falhas dos modelos VaR

\begin{tabular}{|c|c|c|c|c|c|c|}
\hline & \multicolumn{3}{|c|}{ Normal } & \multicolumn{3}{|c|}{ t-student } \\
\hline & 0,005 & 0,01 & 0,05 & 0,005 & 0,01 & 0,05 \\
\hline VECH & 0,00898 & 0,01485 & 0,04696 & 0,01209 & 0,02383 & 0,06457 \\
\hline BEKK & 0,01174 & 0,01865 & 0,04696 & 0,00863 & 0,01623 & 0,05387 \\
\hline CCC & 0,00932 & 0,01588 & 0,04938 & 0,00760 & 0,01278 & 0,05283 \\
\hline TVECH & 0,00967 & 0,01588 & 0,04903 & 0,00311 & 0,00725 & 0,04523 \\
\hline TBEKK & 0,00898 & 0,01519 & 0,05007 & 0,00829 & 0,01657 & 0,05559 \\
\hline TCCC & 0,00967 & 0,01588 & 0,05007 & 0,00656 & 0,01140 & 0,05283 \\
\hline VECH-TVE & 0,00276 & 0,00898 & 0,05007 & 0,00345 & 0,00829 & 0,05007 \\
\hline BECK-TVE & 0,00311 & 0,00794 & 0,05007 & 0,00276 & 0,00760 & 0,05007 \\
\hline CCC-TVE & 0,00311 & 0,00829 & 0,05007 & 0,00345 & 0,00794 & 0,05007 \\
\hline TVECH-TVE & 0,00345 & 0,00863 & 0,05007 & 0,00345 & 0,00863 & 0,05007 \\
\hline TBEKK-TVE & 0,00345 & 0,00863 & 0,05007 & 0,00311 & 0,00829 & 0,05007 \\
\hline TCCC-TVE & 0,00276 & 0,00794 & 0,05007 & 0,00345 & 0,00794 & 0,05007 \\
\hline
\end{tabular}

Notas: as estimativas VaR, apresentadas na tabela, foram obtidas a partir da matriz de variância-covariância de modelos GARCH sem efeito assimétrico (VECH, BEKK, CCC), com efeito assimétrico (TVECH, TBEKK e TCC) e com a TVE, considerando os intervalos de confiança de $99,5 \%(0,5), 99 \%$ (1) e $95 \%$ (5).

Fonte: Do autor.

Após a estimação dos diversos modelos VaR, foram aplicados os procedimentos de Backtesting, de modo a concluir acerca da capacidade desses modelos para gerir adequadamente o risco de mercado dos mercados bolsistas. Em primeiro lugar, foi considerado o teste de cobertura incondicional ou teste de Kupiec, que é um desenvolvimento da taxa de falhas, definido pelo Comitê de Basileia, para a supervisão bancária. Em segundo lugar, foi aplicado o teste de independência para 
testar se as exceçôes são IID. Por último, aplicou-se o teste de cobertura condicional, ou teste de Christoffersen. Os resultados dos testes de Backtesting são apresentados nas Tabelas 6 a 13.

No teste de cobertura incondicional e relativamente aos dois quantis menores, a distribuição t-student apresentou melhor performance que a normal. Em ambas as distribuições, a performance das estimativas melhorou no nível de confiança mais elevado, com níveis de aceitação global dos modelos de $100 \%$ e de $92 \%$, nas distribuiçôes normal e t-student, respetivamente. Dos modelos que não recorreram à TVE, o modelo TCCC, baseado na distribuição t-student, foi o que apresentou melhor desempenho, sendo aceite para todos os níveis de confiança. Em sentido contrário, o modelo VECH (t-student) foi rejeitado para os três quantis. Quanto aos modelos suportados na TVE, todos passaram no teste de Kupiec, nos três níveis de confiança. As especificaçôes da matriz de variâncias-covariâncias não rejeitaram a hipótese nula do teste, suportando a ideia de superioridade da metodologia assente na distribuição generalizada de Pareto, face às metodologias baseadas nas outras duas distribuiçôes.

TABELA 6 - Resultados do Backtesting - modelo GARCH e pressuposto de distribuição normal

\begin{tabular}{|c|c|c|c|c|c|c|c|c|c|}
\hline & $\begin{array}{l}\text { VECH } \\
(0,5 \%)\end{array}$ & $\begin{array}{c}\text { VECH } \\
(1 \%)\end{array}$ & $\begin{array}{c}\text { VECH } \\
(5 \%)\end{array}$ & $\begin{array}{l}\text { BEKK } \\
(0,5 \%)\end{array}$ & $\begin{array}{c}\text { BEKK } \\
(1 \%)\end{array}$ & $\begin{array}{c}\text { BEKK } \\
(5 \%)\end{array}$ & $\begin{array}{c}\text { CCC } \\
(0,5 \%)\end{array}$ & $\begin{array}{l}\text { CCC } \\
(1 \%)\end{array}$ & $\begin{array}{l}\text { CCC } \\
(5 \%)\end{array}$ \\
\hline $\mathrm{T}_{0}$ & 2870 & 2853 & 2760 & 2862 & 2842 & 2760 & 2869 & 2850 & 2753 \\
\hline $\mathrm{T}_{1}$ & 26 & 43 & 136 & 34 & 54 & 136 & 27 & 46 & 143 \\
\hline $\mathrm{T}_{00}$ & 2844 & 2810 & 2636 & 2830 & 2793 & 2642 & 2842 & 2805 & 2625 \\
\hline $\mathrm{T}_{01}$ & 26 & 43 & 124 & 32 & 49 & 118 & 27 & 45 & 128 \\
\hline $\mathrm{T}_{10}$ & 26 & 43 & 124 & 32 & 49 & 118 & 27 & 45 & 128 \\
\hline $\mathrm{T}_{11}$ & 0 & 0 & 12 & 2 & 5 & 18 & 0 & 1 & 15 \\
\hline $\mathrm{p}$ & 0,009 & 0,015 & 0,047 & 0,012 & 0,019 & 0,047 & 0,009 & 0,016 & 0,049 \\
\hline $\mathrm{p}_{01}$ & 0,009 & 0,015 & 0,045 & 0,011 & 0,017 & 0,043 & 0,009 & 0,016 & 0,046 \\
\hline $\mathrm{p}_{11}$ & 0,000 & 0,000 & 0,088 & 0,059 & 0,093 & 0,132 & 0,000 & 0,022 & 0,105 \\
\hline \multirow{2}{*}{$\mathrm{LR}_{\mathrm{uc}}$} & 7,443 & 5,983 & 0,574 & 19,137 & 17,431 & 0,574 & 8,660 & 8,592 & 0,024 \\
\hline & $(0,006)$ & $(0,014)$ & $(0,449)$ & $(0,000)$ & $(0,000)$ & $(0,449)$ & $(0,003)$ & $(0,003)$ & $(0,878)$ \\
\hline \multirow{2}{*}{$\mathrm{LR}_{\text {ind }}$} & 0,471 & 1,296 & 4,415 & 18,613 & 8,663 & 20,192 & 0,508 & 0,092 & 7,696 \\
\hline & $(0,492)$ & $(0,255)$ & $(0,036)$ & $(0,000)$ & $(0,003)$ & $(0,000)$ & $(0,476)$ & $(0,761)$ & $(0,006)$ \\
\hline \multirow{2}{*}{$\mathrm{LR}_{c c}$} & 7,914 & 7,280 & 4,989 & 37,750 & 26,093 & 20,766 & 9,168 & 8,684 & 7,719 \\
\hline & $(0,019)$ & $(0,026)$ & $(0,083)$ & $(0,000)$ & $(0,000)$ & $(0,000)$ & $(0,010)$ & $(0,013)$ & $(0,021)$ \\
\hline
\end{tabular}

Notas: Esta tabela apresenta os resultados dos testes de cobertura incondicional (LRuc), independência (LRind) e cobertura condicional (LRcc), da carteira composta pelos 12 índices e as estimativas VaR, obtidas a partir da matriz de variânciacovariância dos modelos GARCH-VECH, GARCH-BEKK e GARCH-CCC, supondo que as rentabilidades são descritas pela distribuição normal e considerando os intervalos de confiança de 99,5\% (0,5), 99\% (1) e 95\% (5). Os valores entre parênteses dizem respeito à probabilidade de cada um dos testes.

Fonte: Do autor. 
TABELA 7 - Resultados do Backtesting - modelo GARCH e pressuposto de distribuição t-student.

\begin{tabular}{lccccccccc}
\hline & $\mathbf{V E C H}$ & $\mathbf{V E C H}$ & $\mathbf{V E C H}$ & $\mathbf{B E K K}$ & $\mathbf{B E K K}$ & $\mathbf{B E K K}$ & $\mathbf{C C C}$ & $\mathbf{C C C}$ & $\mathbf{C C C}$ \\
& $(\mathbf{0 , 5} \mathbf{)}$ & $(\mathbf{1 \% )}$ & $\mathbf{( 5 \% )}$ & $\mathbf{( 0 , 5 \% )}$ & $\mathbf{( 1 \% )}$ & $\mathbf{5 \% )}$ & $\mathbf{( 0 , 5 \% )}$ & $\mathbf{( 1 \% )}$ & $\mathbf{( 5 \% )}$ \\
\hline $\mathrm{T}_{0}$ & 2861 & 2827 & 2709 & 2871 & 2849 & 2740 & 2874 & 2859 & 2743 \\
$\mathrm{~T}_{1}$ & 35 & 69 & 187 & 25 & 47 & 156 & 22 & 37 & 153 \\
$\mathrm{~T}_{00}$ & 2828 & 2762 & 2547 & 2847 & 2807 & 2607 & 2852 & 2824 & 2606 \\
$\mathrm{~T}_{01}$ & 33 & 65 & 162 & 24 & 42 & 133 & 22 & 35 & 137 \\
$\mathrm{~T}_{10}$ & 33 & 65 & 162 & 24 & 42 & 133 & 22 & 35 & 137 \\
$\mathrm{~T}_{11}$ & 2 & 4 & 25 & 1 & 5 & 23 & 0 & 2 & 16 \\
$\mathrm{p}$ & 0,012 & 0,024 & 0,065 & 0,009 & 0,016 & 0,054 & 0,008 & 0,013 & 0,053 \\
$\mathrm{P}_{01}$ & 0,012 & 0,023 & 0,060 & 0,008 & 0,015 & 0,049 & 0,008 & 0,012 & 0,050 \\
$\mathrm{P}_{11}$ & 0,057 & 0,058 & 0,134 & 0,040 & 0,106 & 0,147 & 0,000 & 0,054 & 0,105 \\
$\mathrm{LR}_{\text {uc }}$ & 20,887 & 40,292 & 11,903 & 6,304 & 9,552 & 0,890 & 3,384 & 2,073 & 0,480 \\
& $(0,000)$ & $(0,000)$ & $(0,001)$ & $(0,012)$ & $(0,002)$ & $(0,345)$ & $(0,066)$ & $(0,150)$ & $(0,488)$ \\
$\mathrm{LR}_{\text {ind }}$ & 3,207 & 2,570 & 12,562 & 1,549 & 11,136 & 20,192 & 0,337 & 2,845 & 6,921 \\
& $(0,073)$ & $(0,109)$ & $(0,000)$ & $(0,213)$ & $(0,001)$ & $(0,000)$ & $(0,562)$ & $(0,092)$ & $(0,009)$ \\
$\mathrm{LR}_{\text {cc }}$ & 24,094 & 42,862 & 24,465 & 7,852 & 20,687 & 21,082 & 3,721 & 4,918 & 7,402 \\
\hline & $(0,000)$ & $(0,000)$ & $(0,000)$ & $(0,020)$ & $(0,000)$ & $(0,000)$ & $(0,156)$ & $(0,086)$ & $(0,025)$ \\
\hline
\end{tabular}

Notas: Esta tabela apresenta os resultados dos testes de cobertura incondicional (LRuc), independência (LRind) e cobertura condicional (LRcc), da carteira composta pelos doze índices, às estimativas VaR, obtidas a partir da matriz de variânciacovariância dos modelos GARCH-VECH, GARCH-BEKK e GARCH-CCC, supondo que as rentabilidades são descritas pela distribuição t-Student e considerando os intervalos de confiança de 99,5\% (0,5), 99\% (1) e 95\% (5). Os valores entre parêntesis dizem respeito à probabilidade de cada um dos testes.

Fonte: Do autor.

TABELA 8 - Resultados do Backtesting - modelo TGARCH e pressuposto de distribuição normal

\begin{tabular}{lccccccccc}
\hline & $\mathbf{V E C H}$ & $\mathbf{V E C H}$ & $\mathbf{V E C H}$ & $\mathbf{B E K K}$ & $\mathbf{B E K K}$ & $\mathbf{B E K K}$ & $\mathbf{C C C}$ & $\mathbf{C C C}$ & $\mathbf{C C C}$ \\
& $(\mathbf{0 , 5} \mathbf{)}$ & $(\mathbf{1 \% )}$ & $\mathbf{( 5 \% )}$ & $\mathbf{( 0 , 5 \% )}$ & $\mathbf{( 1 \% )}$ & $\mathbf{( 5 \% )}$ & $\mathbf{( 0 , 5 \% )}$ & $\mathbf{( 1 \% )}$ & $\mathbf{( 5 \% )}$ \\
\hline $\mathrm{T}_{0}$ & 2868 & 2850 & 2754 & 2870 & 2852 & 2751 & 2868 & 2850 & 2751 \\
$\mathrm{~T}_{1}$ & 28 & 46 & 142 & 26 & 44 & 145 & 28 & 46 & 145 \\
$\mathrm{~T}_{00}$ & 2840 & 2804 & 2626 & 2844 & 2809 & 2624 & 2840 & 2804 & 2619 \\
$\mathrm{~T}_{01}$ & 28 & 46 & 128 & 26 & 43 & 127 & 28 & 46 & 132 \\
$\mathrm{~T}_{10}$ & 28 & 46 & 128 & 26 & 43 & 127 & 28 & 46 & 132 \\
$\mathrm{~T}_{11}$ & 0 & 0 & 14 & 0 & 1 & 18 & 0 & 0 & 13 \\
$\mathrm{p}$ & 0,010 & 0,016 & 0,049 & 0,009 & 0,015 & 0,050 & 0,010 & 0,016 & 0,050 \\
$\mathrm{P}_{01}$ & 0,010 & 0,016 & 0,046 & 0,009 & 0,015 & 0,046 & 0,010 & 0,016 & 0,048 \\
$\mathrm{P}_{11}$ & 0,000 & 0,000 & 0,099 & 0,000 & 0,023 & 0,124 & 0,000 & 0,000 & 0,090 \\
$\mathrm{LR}_{\text {uc }}$ & 9,952 & 8,592 & 0,057 & 7,443 & 6,807 & 0,000 & 9,952 & 8,592 & 0,000 \\
& $(0,002)$ & $(0,003)$ & $(0,811)$ & $(0,006)$ & $(0,009)$ & $(0,986)$ & $(0,002)$ & $(0,003)$ & $(0,986)$ \\
$\mathrm{LR}_{\text {ind }}$ & 0,547 & 1,485 & 6,249 & 0,471 & 0,148 & 12,972 & 0,547 & 1,485 & 4,165 \\
& $(0,460)$ & $(0,223)$ & $(0,012)$ & $(0,492)$ & $(0,701)$ & $(0,000)$ & $(0,460)$ & $(0,223)$ & $(0,041)$ \\
$\mathrm{LR}_{\text {cc }}$ & 10,499 & 10,077 & 6,306 & 7,914 & 6,955 & 12,972 & 10,499 & 10,077 & 4,165 \\
\hline & $(0,005)$ & $(0,006)$ & $(0,043)$ & $(0,019)$ & $(0,031)$ & $(0,002)$ & $(0,005)$ & $(0,006)$ & $(0,125)$ \\
\hline
\end{tabular}

Notas: Esta tabela apresenta os resultados dos testes de cobertura incondicional (LRuc), independência (LRind) e cobertura condicional (LRcc) da carteira composta pelos 12 índices as estimativas VaR, obtidas a partir da matriz de variância-covariância dos modelos Threshold GARCH (VECH, BEKK e CCC), supondo que as rentabilidades são descritas pela distribuição normal e considerando os intervalos de confiança de 99,5\% (0,5), 99\% (1) e 95\% (5). Os valores entre parênteses dizem respeito à probabilidade de cada um dos testes.

Fonte: Do autor. 
Modelos Multivariados na Previsão do Valor em Risco de Carteiras de Investimento: da crise das empresas tecnológicas à crise financeira global

TABELA 9 - Resultados do Backtesting - modelo TGARCH e pressuposto de distribuição t-student

\begin{tabular}{|c|c|c|c|c|c|c|c|c|c|}
\hline & $\begin{array}{l}\text { VECH } \\
(0,5 \%)\end{array}$ & $\begin{array}{c}\text { VECH } \\
(1 \%)\end{array}$ & $\begin{array}{c}\text { VECH } \\
(5 \%)\end{array}$ & $\begin{array}{l}\text { BEKK } \\
(0,5 \%)\end{array}$ & $\begin{array}{c}\text { BEKK } \\
(1 \%)\end{array}$ & $\begin{array}{c}\text { BEKK } \\
(5 \%)\end{array}$ & $\begin{array}{c}\text { CCC } \\
(0,5 \%)\end{array}$ & $\begin{array}{l}\text { CCC } \\
(1 \%)\end{array}$ & $\begin{array}{l}\text { CCC } \\
(5 \%)\end{array}$ \\
\hline $\mathrm{T}_{0}$ & 2887 & 2875 & 2765 & 2872 & 2848 & 2735 & 2877 & 2863 & 2743 \\
\hline $\mathrm{T}_{1}$ & 9 & 21 & 131 & 24 & 48 & 161 & 19 & 33 & 153 \\
\hline $\mathrm{T}_{00}$ & 2878 & 2854 & 2645 & 2848 & 2805 & 2596 & 2858 & 2830 & 2606 \\
\hline $\mathrm{T}_{01}$ & 9 & 21 & 120 & 24 & 43 & 139 & 19 & 33 & 137 \\
\hline $\mathrm{T}_{10}$ & 9 & 21 & 120 & 24 & 43 & 139 & 19 & 33 & 137 \\
\hline $\mathrm{T}_{11}$ & 0 & 0 & 11 & 0 & 5 & 22 & 0 & 0 & 16 \\
\hline $\mathrm{p}$ & 0,003 & 0,007 & 0,045 & 0,008 & 0,017 & 0,056 & 0,007 & 0,011 & 0,053 \\
\hline $\mathrm{p}_{01}$ & 0,003 & 0,007 & 0,043 & 0,008 & 0,015 & 0,051 & 0,007 & 0,012 & 0,050 \\
\hline $\mathrm{p}_{11}$ & 0,000 & 0,000 & 0,084 & 0,000 & 0,104 & 0,137 & 0,000 & 0,000 & 0,105 \\
\hline \multirow{2}{*}{$\mathrm{LR}_{\mathrm{uc}}$} & 2,411 & 2,444 & 1,428 & 5,245 & 10,554 & 1,844 & 1,291 & 0,545 & 0,480 \\
\hline & $(0,121)$ & $(0,118)$ & $(0,232)$ & $(0,022)$ & $(0,001)$ & $(0,174)$ & $(0,256)$ & $(0,460)$ & $(0,488)$ \\
\hline \multirow{2}{*}{$\mathrm{LR}_{\text {ind }}$} & 0,056 & 0,307 & 3,888 & 0,401 & 10,751 & 15,844 & 0,251 & 0,761 & 6,921 \\
\hline & $(0,813)$ & $(0,580)$ & $(0,049)$ & $(0,527)$ & $(0,001)$ & $(0,000)$ & $(0,616)$ & $(0,383)$ & $(0,009)$ \\
\hline \multirow{2}{*}{$\mathrm{LR}_{\mathrm{cc}}$} & 2,467 & 2,750 & 5,316 & 5,646 & 21,305 & 17,688 & 1,542 & 1,306 & 7,402 \\
\hline & $(0,291)$ & $(0,253)$ & $(0,070)$ & $(0,059)$ & $(0,000)$ & $(0,000)$ & $(0,463)$ & $(0,521)$ & $(0,025)$ \\
\hline
\end{tabular}

Notas: Esta tabela apresenta os resultados dos testes de cobertura incondicional (LRuc), independência (LRind) e cobertura condicional (LRcc) da carteira composta pelos 12 índices e às estimativas VaR, obtidas a partir da matriz de variânciacovariância dos modelos Threshold GARCH (VECH, BEKK e CCC), supondo que as rentabilidades são descritas pela distribuição t-student e considerando os intervalos de confiança de 99,5\% (0,5), 99\% (1) e 95\% (5). Os valores entre parênteses dizem respeito à probabilidade de cada um dos testes.

Fonte: Do autor.

TABELA 10 - Resultados do Backtesting - modelo GARCH-TVE e pressuposto de distribuição normal

\begin{tabular}{|c|c|c|c|c|c|c|c|c|c|}
\hline & $\begin{array}{l}\text { VECH } \\
(0,5 \%)\end{array}$ & $\begin{array}{c}\text { VECH } \\
(1 \%)\end{array}$ & $\begin{array}{c}\text { VECH } \\
(5 \%)\end{array}$ & $\begin{array}{l}\text { BEKK } \\
(0,5 \%)\end{array}$ & $\begin{array}{c}\text { BEKK } \\
(1 \%)\end{array}$ & $\begin{array}{c}\text { BEKK } \\
(5 \%)\end{array}$ & $\begin{array}{c}\text { CCC } \\
(0,5 \%)\end{array}$ & $\begin{array}{l}\text { CCC } \\
(1 \%)\end{array}$ & $\begin{array}{l}\text { CCC } \\
(5 \%)\end{array}$ \\
\hline $\mathrm{T}_{0}$ & 2888 & 2870 & 2751 & 2887 & 2873 & 2751 & 2887 & 2872 & 2751 \\
\hline $\mathrm{T}_{1}$ & 8 & 26 & 145 & 9 & 23 & 145 & 9 & 24 & 145 \\
\hline $\mathrm{T}_{00}$ & 2880 & 2844 & 2621 & 2878 & 2850 & 2625 & 2878 & 2848 & 2622 \\
\hline $\mathrm{T}_{01}$ & 8 & 26 & 130 & 9 & 23 & 126 & 9 & 24 & 129 \\
\hline $\mathrm{T}_{10}$ & 8 & 26 & 130 & 9 & 23 & 126 & 9 & 24 & 129 \\
\hline $\mathrm{T}_{11}$ & 0 & 0 & 15 & 0 & 0 & 19 & 0 & 0 & 16 \\
\hline $\mathrm{p}$ & 0,003 & 0,009 & 0,050 & 0,003 & 0,008 & 0,050 & 0,003 & 0,008 & 0,050 \\
\hline $\mathrm{p}_{01}$ & 0,003 & 0,009 & 0,047 & 0,003 & 0,008 & 0,046 & 0,003 & 0,008 & 0,047 \\
\hline $\mathrm{p}_{11}$ & 0,000 & 0,000 & 0,103 & 0,000 & 0,000 & 0,131 & 0,000 & 0,000 & 0,110 \\
\hline \multirow{2}{*}{$\mathrm{LR}_{\mathrm{uc}}$} & 3,481 & 0,316 & 0,000 & 2,411 & 1,333 & 0,000 & 2,411 & 0,911 & 0,000 \\
\hline & $(0,062)$ & $(0,574)$ & $(0,986)$ & $(0,121)$ & $(0,248)$ & $(0,986)$ & $(0,121)$ & $(0,340)$ & $(0,986)$ \\
\hline \multirow{2}{*}{$\mathrm{LR}_{\text {ind }}$} & 0,044 & 0,471 & 7,200 & 0,056 & 0,368 & 15,192 & 0,056 & 0,401 & 8,969 \\
\hline & $(0,833)$ & $(0,492)$ & $(0,007)$ & $(0,813)$ & $(0,544)$ & $(0,000)$ & $(0,813)$ & $(0,527)$ & $(0,003)$ \\
\hline \multirow{2}{*}{$\mathrm{LR}_{\mathrm{cc}}$} & 3,526 & 0,788 & 7,200 & 2,467 & 1,701 & 15,192 & 2,467 & 1,312 & 8,970 \\
\hline & $(0,172)$ & $(0,675)$ & $(0,027)$ & $(0,291)$ & $(0,427)$ & $(0,001)$ & $(0,291)$ & $(0,519)$ & $(0,011)$ \\
\hline
\end{tabular}

Notas: Esta tabela apresenta os resultados dos testes de cobertura incondicional (LRuc), independência (LRind) e cobertura condicional (LRcc) da carteira composta pelos 12 índices e as estimativas VaR, obtidas a partir da matriz de variânciacovariância dos modelos GARCH (VECH, BEKK e CCC) e da TVE, supondo que as rentabilidades são descritas pela distribuição normal e considerando os intervalos de confiança de 99,5\% (0,5), 99\% (1) e 95\% (5). Os valores entre parênteses dizem respeito à probabilidade de cada um dos testes.

Fonte: Do autor. 
TABELA 11 - Resultados do Backtesting - modelo GARCH-TVE e pressuposto de distribuição t-student

\begin{tabular}{|c|c|c|c|c|c|c|c|c|c|}
\hline & $\begin{array}{l}\text { VECH } \\
(0,5 \%)\end{array}$ & $\begin{array}{c}\text { VECH } \\
(1 \%)\end{array}$ & $\begin{array}{c}\text { VECH } \\
(5 \%)\end{array}$ & $\begin{array}{l}\text { BEKK } \\
(0,5 \%)\end{array}$ & $\begin{array}{c}\text { BEKK } \\
(1 \%)\end{array}$ & $\begin{array}{c}\text { BEKK } \\
(5 \%)\end{array}$ & $\begin{array}{c}\text { CCC } \\
(0,5 \%)\end{array}$ & $\begin{array}{l}\text { CCC } \\
(1 \%)\end{array}$ & $\begin{array}{l}\text { CCC } \\
(5 \%)\end{array}$ \\
\hline $\mathrm{T}_{0}$ & 2886 & 2872 & 2751 & 2888 & 2874 & 2751 & 2886 & 2873 & 2751 \\
\hline $\mathrm{T}_{1}$ & 10 & 24 & 145 & 8 & 22 & 145 & 10 & 23 & 145 \\
\hline $\mathrm{T}_{00}$ & 2876 & 2848 & 2624 & 2880 & 2852 & 2626 & 2876 & 2850 & 2622 \\
\hline $\mathrm{T}_{01}$ & 10 & 24 & 127 & 8 & 22 & 125 & 10 & 23 & 129 \\
\hline $\mathrm{T}_{10}$ & 10 & 24 & 127 & 8 & 22 & 125 & 10 & 23 & 129 \\
\hline $\mathrm{T}_{11}$ & 0 & 0 & 18 & 0 & 0 & 20 & 0 & 0 & 16 \\
\hline $\mathrm{p}$ & 0,003 & 0,008 & 0,050 & 0,003 & 0,008 & 0,050 & 0,003 & 0,008 & 0,050 \\
\hline $\mathrm{p}_{01}$ & 0,003 & 0,008 & 0,046 & 0,003 & 0,008 & 0,045 & 0,003 & 0,008 & 0,047 \\
\hline $\mathrm{p}_{11}$ & 0,000 & 0,000 & 0,124 & 0,000 & 0,000 & 0,138 & 0,000 & 0,000 & 0,110 \\
\hline \multirow{2}{*}{$\mathrm{LR}_{\mathrm{uc}}$} & 1,563 & 0,911 & 0,000 & 3,481 & 1,842 & 0,000 & 1,563 & 1,333 & 0,000 \\
\hline & $(0,211)$ & $(0,340)$ & $(0,986)$ & $(0,062)$ & $(0,175)$ & $(0,986)$ & $(0,211)$ & $(0,248)$ & $(0,986)$ \\
\hline \multirow{2}{*}{$\mathrm{LR}_{\text {ind }}$} & 0,069 & 0,401 & 12,972 & 0,044 & 0,337 & 17,549 & 0,069 & 0,368 & 8,969 \\
\hline & $(0,792)$ & $(0,527)$ & $(0,000)$ & $(0,833)$ & $(0,562)$ & $(0,000)$ & $(0,792)$ & $(0,544)$ & $(0,003)$ \\
\hline \multirow{2}{*}{$\mathrm{LR}_{\mathrm{cc}}$} & 1,633 & 1,312 & 12,972 & 3,526 & 2,179 & 17,549 & 1,633 & 1,701 & 8,970 \\
\hline & $(0,442)$ & $(0,519)$ & $(0,002)$ & $(0,172)$ & $(0,336)$ & $(0,000)$ & $(0,442)$ & $(0,427)$ & $(0,011)$ \\
\hline
\end{tabular}

Notas: Esta tabela apresenta os resultados dos testes de cobertura incondicional (LRuc), independência (LRind) e cobertura condicional (LRcc) da carteira composta pelos 12 índices, as estimativas VaR, obtidas a partir da matriz de variânciacovariância dos modelos GARCH (VECH, BEKK e CCC) e da TVE, supondo que as rentabilidades são descritas pela distribuição t-student e considerando os intervalos de confiança de 99,5\% (0,5), 99\% (1) e 95\% (5). Os valores entre parênteses dizem respeito à probabilidade de cada um dos testes.

Fonte: Do autor.

TABELA 12 - Resultados do Backtesting - modelo TGARCH-TVE e pressuposto de distribuição normal

\begin{tabular}{lccccccccc}
\hline & $\mathbf{V E C H}$ & $\mathbf{V E C H}$ & $\mathbf{V E C H}$ & $\mathbf{B E K K}$ & $\mathbf{B E K K}$ & $\mathbf{B E K K}$ & $\mathbf{C C C}$ & $\mathbf{C C C}$ & $\mathbf{C C C}$ \\
& $(\mathbf{0 , 5} \mathbf{)}$ & $\mathbf{( 1 \% )}$ & $\mathbf{( 5 \% )}$ & $\mathbf{( 0 , 5 \% )}$ & $\mathbf{( 1 \% )}$ & $\mathbf{( 5 \% )}$ & $\mathbf{( 0 , 5 \% )}$ & $\mathbf{( 1 \% )}$ & $\mathbf{( 5 \% )}$ \\
\hline $\mathrm{T}_{0}$ & 2886 & 2871 & 2751 & 2886 & 2871 & 2751 & 2888 & 2873 & 2751 \\
$\mathrm{~T}_{1}$ & 10 & 25 & 145 & 10 & 25 & 145 & 8 & 23 & 145 \\
$\mathrm{~T}_{00}$ & 2876 & 2846 & 2618 & 2876 & 2846 & 2624 & 2880 & 2850 & 2619 \\
$\mathrm{~T}_{01}$ & 10 & 25 & 133 & 10 & 25 & 127 & 8 & 23 & 132 \\
$\mathrm{~T}_{10}$ & 10 & 25 & 133 & 10 & 25 & 127 & 8 & 23 & 132 \\
$\mathrm{~T}_{11}$ & 0 & 0 & 12 & 0 & 0 & 18 & 0 & 0 & 13 \\
$\mathrm{p}$ & 0,003 & 0,009 & 0,050 & 0,003 & 0,009 & 0,050 & 0,003 & 0,008 & 0,050 \\
$\mathrm{P}_{01}$ & 0,003 & 0,009 & 0,048 & 0,003 & 0,009 & 0,046 & 0,003 & 0,008 & 0,048 \\
$\mathrm{P}_{11}$ & 0,000 & 0,000 & 0,083 & 0,000 & 0,000 & 0,124 & 0,000 & 0,000 & 0,090 \\
$\mathrm{LR}_{\text {uc }}$ & 1,563 & 0,573 & 0,000 & 1,563 & 0,573 & 0,000 & 3,481 & 1,333 & 0,000 \\
& $(0,211)$ & $(0,449)$ & $(0,986)$ & $(0,211)$ & $(0,449)$ & $(0,986)$ & $(0,062)$ & $(0,248)$ & $(0,986)$ \\
$\mathrm{LR}_{\text {ind }}$ & 0,069 & 0,435 & 2,919 & 0,069 & 0,435 & 12,972 & 0,044 & 0,368 & 4,165 \\
& $(0,792)$ & $(0,509)$ & $(0,088)$ & $(0,792)$ & $(0,509)$ & $(0,000)$ & $(0,833)$ & $(0,544)$ & $(0,041)$ \\
$\mathrm{LR}_{\text {cc }}$ & 1,633 & 1,009 & 2,920 & 1,633 & 1,009 & 12,972 & 3,526 & 1,701 & 4,165 \\
\hline & $(0,442)$ & $(0,604)$ & $(0,232)$ & $(0,442)$ & $(0,604)$ & $(0,002)$ & $(0,172)$ & $(0,427)$ & $(0,125)$ \\
\hline
\end{tabular}

Notas: Esta tabela apresenta os resultados dos testes de cobertura incondicional (LRuc), independência (LRind) e cobertura condicional (LRcc) da carteira composta pelos 12 índices e às estimativas VaR, obtidas a partir da matriz de variânciacovariância dos modelos Threshold GARCH (VECH, BEKK e CCC) e da TVE, supondo que as rentabilidades são descritas pela distribuição normal e considerando os intervalos de confiança de 99,5\% (0,5), 99\% (1) e 95\% (5). Os valores entre parênteses dizem respeito à probabilidade de cada um dos testes.

Fonte: Do autor. 
Modelos Multivariados na Previsão do Valor em Risco de Carteiras de Investimento: da crise das empresas tecnológicas à crise financeira global

TABELA 13 - Resultados do Backtesting - modelo TGARCH-TVE e pressuposto de distribuição t-student

\begin{tabular}{|c|c|c|c|c|c|c|c|c|c|}
\hline & $\begin{array}{l}\text { VECH } \\
(0,5 \%)\end{array}$ & $\begin{array}{c}\text { VECH } \\
(1 \%)\end{array}$ & $\begin{array}{c}\text { VECH } \\
(5 \%)\end{array}$ & $\begin{array}{l}\text { BEKK } \\
(0,5 \%)\end{array}$ & $\begin{array}{c}\text { BEKK } \\
(1 \%)\end{array}$ & $\begin{array}{c}\text { BEKK } \\
(5 \%)\end{array}$ & $\begin{array}{c}\text { CCC } \\
(0,5 \%)\end{array}$ & CCC (1\%) & $\begin{array}{l}\text { CCC } \\
(5 \%)\end{array}$ \\
\hline $\mathrm{T}_{0}$ & 2886 & 2871 & 2751 & 2887 & 2872 & 2751 & 2886 & 2873 & 2751 \\
\hline $\mathrm{T}_{1}$ & 10 & 25 & 145 & 9 & 24 & 145 & 10 & 23 & 145 \\
\hline $\mathrm{T}_{00}$ & 2876 & 2846 & 2618 & 2878 & 2848 & 2624 & 2876 & 2850 & 2621 \\
\hline $\mathrm{T}_{01}$ & 10 & 25 & 133 & 9 & 24 & 127 & 10 & 23 & 130 \\
\hline $\mathrm{T}_{10}$ & 10 & 25 & 133 & 9 & 24 & 127 & 10 & 23 & 130 \\
\hline $\mathrm{T}_{11}$ & 0 & 0 & 12 & 0 & 0 & 18 & 0 & 0 & 15 \\
\hline $\mathrm{p}$ & 0,003 & 0,009 & 0,050 & 0,003 & 0,008 & 0,050 & 0,003 & 0,008 & 0,050 \\
\hline $\mathrm{p}_{01}$ & 0,003 & 0,009 & 0,048 & 0,003 & 0,008 & 0,046 & 0,003 & 0,008 & 0,047 \\
\hline $\mathrm{p}_{11}$ & 0,000 & 0,000 & 0,083 & 0,000 & 0,000 & 0,124 & 0,000 & 0,000 & 0,103 \\
\hline \multirow{2}{*}{$\mathrm{LR}_{\mathrm{uc}}$} & 1,563 & 0,573 & 0,000 & 2,411 & 0,911 & 0,000 & 1,563 & 1,333 & 0,000 \\
\hline & $(0,211)$ & $(0,449)$ & $(0,986)$ & $(0,121)$ & $(0,340)$ & $(0,986)$ & $(0,211)$ & $(0,248)$ & $(0,986)$ \\
\hline \multirow{2}{*}{$\mathrm{LR}_{\text {ind }}$} & 0,069 & 0,435 & 2,919 & 0,056 & 0,401 & 12,972 & 0,069 & 0,368 & 7,200 \\
\hline & $(0,792)$ & $(0,509)$ & $(0,088)$ & $(0,813)$ & $(0,527)$ & $(0,000)$ & $(0,792)$ & $(0,544)$ & $(0,007)$ \\
\hline \multirow{2}{*}{$\mathrm{LR}_{\mathrm{cc}}$} & 1,633 & 1,009 & 2,920 & 2,467 & 1,312 & 12,972 & 1,633 & 1,701 & 7,200 \\
\hline & $(0,442)$ & $(0,604)$ & $(0,232)$ & $(0,291)$ & $(0,519)$ & $(0,002)$ & $(0,442)$ & $(0,427)$ & $(0,027)$ \\
\hline
\end{tabular}

Notas: Esta tabela apresenta os resultados dos testes de cobertura incondicional (LRuc), independência (LRind) e cobertura condicional (LRcc) da carteira composta pelos 12 índices e as estimativas VaR, obtidas a partir da matriz de variânciacovariância dos modelos Threshold GARCH (VECH, BEKK e CCC) e da TVE, supondo que as rentabilidades são descritas pela distribuição t-Student e considerando os intervalos de confiança de 99,5\% (0,5), 99\% (1) e 95\% (5). Os valores entre parênteses dizem respeito à probabilidade de cada um dos testes.

Fonte: Do autor.

A análise à independência temporal das exceções revelou que, em geral, os modelos registraram uma boa performance nos dois quantis menores. Apenas o modelo BEKK evidenciou dificuldade em detectar as exceções em cluster, em especial na distribuição normal. Relativamente ao quantil mais elevado, os modelos demonstraram uma clara dificuldade na identificação da independência, tendo o modelo TVECH-TVE sido o único a revelar capacidade para captar o efeito de independência, patente em ambas as distribuições. Por vez, a comparação entre as duas distribuiçôes traduz-se em resultados muito idênticos, com a distribuição normal a validar $91,7 \%, 91,7 \%$ e $8,3 \%$ dos modelos, nos quantis $0,5 \%, 1 \%$ e $5 \%$, respectivamente, ao passo que a distribuição t-student contribui para validar $100 \%, 83,3 \%$ e $8,3 \%$ dos modelos em cada um dos quantis referidos. Esses resultados permitem a conclusão de que nenhuma das distribuiçôes tem superioridade em relação à outra, pelo que a capacidade dos modelos para testar a propriedade de as exceções serem IID não parece depender do tipo de distribuição considerado.
O teste de cobertura condicional, que conjuga os efeitos de cobertura incondicional e de independência, evidenciou a superioridade dos modelos baseados na distribuição t-student face aos baseados na distribuiçáo normal, nos níveis de confiança de $0,5 \%$ e de $1 \%$, sendo aceites em $83 \%$ e $75 \%$ dos casos, para os dois níveis de confiança anteriores, respectivamente, face aos $50 \%$ de aceitação na distribuição normal. Para o nível de confiança mais elevado, a situação altera-se, com a distribuição normal $(33,3 \%)$ sendo superior à t-student (16,7\%), embora em ambos os casos a performance demonstrada tenha sido bastante limitada.

Vários foram os diversos modelos a demonstrarem incapacidade como instrumentos de gestão do risco, nos três níveis de confiança, em especial os modelos com pressuposto de distribuição normal (BEKK, CCC, TVECH e TBEKK), o mesmo acontecendo com alguns dos modelos suportados na distribuiçáo t-student, como foi o caso dos modelos VECH e BEKK. Em sentido inverso, os modelos TVECH (t-student), TVECH-TVE (normal e t-student) 
e TCCC-TVE (normal) revelaram performances consistentes nos três quantis. É importante realçar que a TVE conduziu a uma evidente melhoria no desempenho dos modelos VaR, em especial nos dois quantis mais baixos, com todos os modelos inspirados nesta metodologia passando no teste de Christoffersen. Relativamente ao nível de confiança mais elevado, apenas os modelos TVECH e TCCC apresentaram um bom desempenho, o primeiro nas duas distribuiçóes e o segundo na distribuição normal.

Os resultados obtidos sugerem que o elemento mais importante na precisão das estimativas dos modelos VaR é o da utilização da TVE, cuja gênese teórica recorre à distribuição generalizada de Pareto. Essa situação realça a importância dessa abordagem, como fator determinante na performance dos modelos, à semelhança de resultados obtidos por outros autores - por exemplo, Lopez e Walter (2001); em uma segunda ordem de importância, aparece a especificação do modelo multivariado.

Do conjunto de especificaçôes consideradas no presente estudo, o modelo multivariado BEKK foi claramente o que apresentou a pior performance, ao passo que o modelo TVECH-TVE foi o que se destacou positivamente em meio aos restantes, sendo validado em todos os testes e nas duas distribuiçóes, revelando, pois, capacidade para incorporar as condições extremas vividas nos mercados, que marcaram o período considerado no presente estudo, em consequência da ocorrência das crises Dot-Com e financeira global.

\section{CONCLUSÃOEIMPLICAÇÓESFUTURAS}

Neste trabalho foi estudada a adequabilidade das estimativas $\mathrm{VaR}$ às condiçóes de mercado, considerando uma carteira teórica de investimento, composta por 12 índices bolsistas internacionais, no período compreendido entre a crise Dot-Com e a crise financeira global. Para o efeito, partiu-se das matrizes de covariâncias, geradas por meio de diversos modelos multivariados de heterocedasticidade condicionada, estimados de acordo com as distribuiçôes normal e t-student, para prever o VaR, à semelhança das metodologias seguidas em outros trabalhos. De forma diferenciada relativamente a esses trabalhos, foi sugerida, porém, uma nova metodologia, que conjuga os referidos modelos com a teoria dos valores extremos, na condição de metodologia de estimação do VaR, com o objetivo de tentar acomodar convenientemente a elevada turbulência que caracterizou os mercados bolsistas, entre os quais os representativos de estados europeus sob assistência financeira internacional.

A comparação entre os somatórios dos VaR dos diversos índices e o VaR da carteira de investimento permitiu a conclusão de que, em todas as situaçóes, o VaR da carteira é claramente inferior. Esse fato permite a oportunidade de minimização do risco de mercado, quando um investidor aposte em uma estratégia de diversificação internacional. Apesar de a ligação entre os mercados bolsistas ser cada vez maior próxima, uma estratégia de diversificação, que considere um conjunto alargado de mercados, pode constituir uma forma de proteção do investidor face ao risco de mercado.

A aplicação de testes de validação de performance das estimativas $\mathrm{VaR}$ revela que o elemento mais importante na precisão dessas estimativas é o da utilização da TVE, baseada na distribuição generalizada de Pareto. Em segundo plano, em termos de importância, aparece a especificação do modelo multivariado. Do conjunto de especificaçóes consideradas no presente estudo, o modelo multivariado BEKK foi claramente o que apresentou o pior desempenho, ao passo que o modelo TVECH-TVE foi o que se destacou positivamente dos restantes, sendo validado em todos os testes e nas duas distribuiçôes, revelando capacidade para incorporar as condiçôes extremas de mercado, vividas durante o lapso de tempo estudado.

Os resultados obtidos mostram que a metodologia proposta neste trabalho tem capacidade para acomodar a elevada turbulência dos mercados, pelo que pode ser vista como uma opção adequada na gestão do risco de mercado.

Em futuros trabalhos, será interessante recorrer a um modelo de otimização que considere, no mesmo plano, rentabilidades de mercados 
e estimativas de risco, associando os modelos GARCH multivariados à TVE, de modo a obter informação adicional, para os investidores internacionais, acerca de suas alternativas de investimento, tendo em conta as oportunidades proporcionadas por mercados desenvolvidos e emergentes.

\section{REFERÊNCIAS}

ANDREEV, V. O. et al. An application of EVT, GPD and POT methods in the russian stock market (RTS index). Nov. 2009. Working Papers Series. Disponível em: <http:// papers.ssrn.com/ sol3/papers.cfm?abstract_id=1507678>. Acesso em: 12 mar. 2013.

ANGELIDIS, T.; BENOS, A.; DEGIANNAKIS, $S$. The use of GARCH models in VaR estimation. Statistical Methodology, [S. 1.], v.1, n. 1-2, p. 105-128, Dec. 2004.

ASSAF, A. Extreme observations and risk assessment in the equity markets of MENA region: tail measures and Value-at-Risk. International Review of Financial Analysis, Amsterdam, v. 18, n. 3. p. 109-116, June 2009.

BEST, P. ImplementingValue-at-Risk. Chichester: John Wiley \& Sons, 1998.

BOLLERSLEV, T. Modeling the coherence in the short-run nominal exchange rates: a multivariate generalized ARCH model. Review of Economics and Statistics, Cambridge, v. 72, n. 3, p. 498505, Aug. 1990.

; ENGLE, R. F.; WOOLDRIDGE, J. M.

A capital asset pricing model with time-varying covariances. Journal of Political Economy, Chicago, v. 96, n. 1, p. 116-131, Feb. 1988.

CAMPBELL, S. D. A review of backtesting and backtesting procedures. 2005. Finance and Economics Discussion Series, Federal Reserve Board, Washington, D.C. Disponível em: <http://www. federalreserve.gov/pubs/feds/2005/200521/200521 pap. pdf>. Acesso em: 12 mar. 2013.
CAPORIN, M.; MCALEER, M. Robust ranking of multivariate GARCH models by problem dimension. June, 2012. Kyoto University/KIER Working Papers 815.

CHRISTOFFERSEN, P. Elements of financial risk management. Amsterdam: Academic Press, 2003.

DANIELSSON, J.; DE VRIES, C. G. Value-at-Risk and extreme returns. 1997. Discussion Paper 273 LSE Financial Markets Group, London School of Economics.

EMBRECHTS, P.; KLUPPERLBERG, C.; MIKOSCH, T. Modelling extreme events for insurance and finance. Berlin, Germany: Springer, 1997.

ENGLE, R. F. Autoregressive conditional hetereoskedasticity with estimates of the variance of United Kingdom inflation. Econometrica, Oxford, v. 50, n. 4 p. 987-1007, July 1982.

; KRONER, K. F. Multivariate simultaneous generalised GARCH. Econometric Theory, Cambridge, v. 11, n. 1, p. 122-150, Mar. 1995.

ESCH, L.; KIEFFER, R.; LOPEZ, T. Asset and risk management: risk oriented finance. England: Wiley Finance, 2005.

FERREIRA, M. A.; LOPEZ, J. A. Evaluating interest rate covariance models within a Value-at-Risk framework. Journal of Financial Econometrics, Oxford, v. 3, n. 1, p. 126-168, Winter 2005.

GIOT, P.; LAURENT, S. Value-at-risk for long and short trading positions. Journal of Applied Econometrics, Chichester, v. 18, n. 6, p. 641664, Nov./Dec. 2003.

HARRIS, R.; SOLLIS, R. Modelling and forecasting financial time series. New York: Wiley, 2003.

JORION, P. Value at risk: the new benchmark for managing financial risk. 3rd ed. United States: McGraw-Hill, 2007. 
KUPIEC, P. H. Techniques for verifying the accuracy of risk management models. Journal of Derivatives, [S. 1.], v. 3, n. 2, p. 73-84, Winter 1995.

LOPEZ, J. A.; WALTER, C. A. Evaluating covariance matrix forecasts in a value-at-risk framework. Journal of Risk, London, v.3, p. 69-98, Apr. 2001.

MANGANELLI, S.; ENGLE, R. F. Value at risk models in finance. Aug. 2001. European Central Bank, Working Paper No 75, p.1-40.

MARKOWITZ, H. Portfolio selection. Journal of Finance, Malden, v.7, n.1, p. 77-91, Mar. 1952.

MARIMOUTOU, V.; RAGGAD, B.; TRABELSI, A. Extreme value theory and Value at Risk: application to oil market. Energy Economics, Amsterdam, n. 31, p. 519-530, Feb. 2009.

MCNEIL, A. J. Calculating quantile risk measures for financial return series using extreme value theory. 1998. Department Mathematik, ETH Institutional Repository, Zentrum, Zurich.

Extreme value theory for risk managers. Internal modelling and CAD II. London: Risk Books, 1999.

; FREY, R. Estimation of tail-related risk measures for heteroscedastic financial time series: an extreme value approach. Journal of Empirical Finance, Amsterdam, v.7, n. 3-4, p. 271-300, Nov. 2000.
MORIMOTO, T.; KAWASAKI, Y. Empirical comparison of multivariate GARCH models for estimation of intraday Value at Risk. 2008. Disponível em: <http://dx.doi.org/ 10.2139/ssrn>. Acesso em: 02 maio 2013.

NIGUEZ, T. M. Volatility and VaR forecasting in the Madrid Stock Exchange. Spanish Economic Review, Heidelberg, v. 10, p. 169-196, 2008.

REISS, R. D.; THOMAS, M. Statistical analysis of extreme values: with applications to insurance, finance, hydrology and other fields. Basel, Switzerland: Birkhauser-Verlag, 1997.

SCHERRER, W.; RIBARITS, E. On the parameterization of multivariate GARCH models. Econometric Theory, Cambridge, v. 23, n. 3, p.464-484, Jun. 2007.

SO, M. K. P.; YU, P. L. H. Empirical analysis of GARCH models in value at risk estimation. International Financial Markets, Institutions and Money, Binghamton; Amsterdam, v. 16, n. 2, p. 180-197, Apr. 2006.

TSAY, R. S. Analysis of financial time series. New Jersey: John Wiley \& Sons, 2005.

WORLD BANK GROUP. c2014. Disponível em: <http://data.worldbank.org/indicator/ CM.MKT.LCAP.CD>. Acesso em: 02 maio 2013.

WU, P. T.; SHIEH, S. J. Value-at-Risk analysis for long-term interest rate futures: Fat-tail and long memory in return innovations. Journal of Empirical Finance, Amsterdam, v. 14, n. 2, p. 248-259, Mar. 2007. 